\title{
The Effect of Canakinumab Treatment on the Inflammatory Markers in Children with Colchicine-Resistant Familial Mediterranean Fever
}

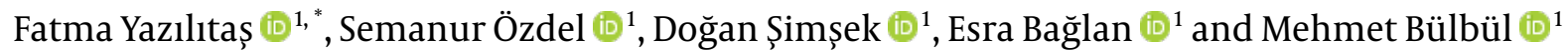 \\ ${ }^{1}$ Ankara Dr. Sami Ulus Maternity and Children Hospital Pediatric Nephrology and Rheumatology Department, Ankara, Turkey \\ "Corresponding author: Ankara Dr. Sami Ulus Maternity and Children Hospital Pediatric Nephrology and Rheumatology Department, Ankara, Turkey. Email: \\ fmeryemesra@yahoo.com
}

Received 2018 September 26; Revised 2019 March 07; Accepted 2019 March 27.

\begin{abstract}
Objectives: This study aimed to determine the reliability of novel markers of treatment response in children with familial Mediterranean fever receiving canakinumab treatment, as compared to conventional inflammatory indexes

Methods: Clinical and laboratory data for 19 colchicine-resistant Familial Mediterranean Fever patients treated with canakinumab were retrospectively reviewed. Patients with systemic diseases other than familial Mediterranean fever and familial Mediterranean fever related complications were excluded from the study.

Results: Following canakinumab treatment there was a non-significant decrease in the neutrophil/ lymphocyte ratio $(\mathrm{P}=0.523)$ and a significant decrease in the platelet/lymphocyte ratio $(\mathrm{P}=0.003)$. There wasn no significant difference in mean platelet volume before and after canakinumab treatment $(\mathrm{P}=0.762)$.

Conclusions: The present findings indicate that the platelet/lymphocyte ratio could be considered a more reliable marker than the neutrophil/lymphocyte ratio of response to canakinumab therapy in patients with colchicine-resistant familial Mediterranean fever.
\end{abstract}

Keywords: Canakinumab, FMF, Inflammatory Markers

\section{Background}

Familial Mediterranean fever (FMF) is an interleukin 1 beta (IL-1 $\beta$ )-dependent disease and is the most common monogenic systemic autoinflammatory disease. FMF is also a hereditary autosomal recessive disorder that has a negative effect on quality of life (QoL) (1). FMF is associated with point mutations in the Mediterranean fever (MEFV) gene, which is located on the short arm of chromosome 16. The MEFV gene encodes the protein pyrin (also referred to as marenostrin) an important modulator of natural immunity (2). Pyrin is part of the inflammasome complex; mutated pyrin leads to excessive neutrophil activation and systemic inflammation due to an increase in the synthesis of IL-1 $\beta$, which plays a major role in the pathogenesis of FMF (3). FMF patients have a high erythrocyte sedimentation rate (ESR), and high C-reactive protein (CRP), serum amyloid A (SAA), and fibrinogen levels during attacks that typically last 1-3 d; these parameters usually return to normal following an attack, but subclinical inflammation can persist in some patients (4). Chronic subclinical inflammation can lead to the development and progression of secondary amyloidosis, the most important and destructive complication of FMF (5).

Canakinumab is a humanized monoclonal antibody against the interleukin IL-1 that binds to IL-1 $\beta$ with high specificity, reducing its bioactivity, and is used to treat FMF associated amyloidosis and colchicine-resistant FMF (crFMF) (6). Recent research has shown that mean platelet volume (MPV), the neutrophil/lymphocyte ratio (NLR), and the platelet/lymphocyte ratio (PLR) are parameters that can be used to monitor disease activity in patients with chronic systemic inflammatory diseases such as FMF (4, 710).

An increase in the CRP level and ESR in the presence of infection may not reliably indicate the degree of inflammation in FMF patients, especially in those with severe infection due to canakinumab treatment. MPV, the NLR, and the PLR were recently reported to be parameters useful for monitoring disease activity and complications in FMF patients; however, to the best of our knowledge no study has evaluated the effect of canakinumab treatment on these parameters. Clinicians treating FMF patients require parameters that can be used as indicators of disease activity which are less expensive, and more readily available and re- 
liable than the CRP level and ESR. MPV, NLR, and PLR all are less expensive, easier to use, and more readily available parameters than the CRP level and ESR and can be measured via a routine blood count.

\section{Objectives}

The aim of the present study was to determine the usefulness of MPV, NLR, and PLR, as indicators of acute phase response (also may be referred to as conventional inflammatory indexes), and as markers of response to canakinumab treatment in patients with cr-FMF.

\section{Methods}

The records of FMF patients treated with canakinumab at our hospital's Department of Pediatric Nephrology and Rheumatology between 2012 and 2018 were retrospectively reviewed. With the permission of our institution, we applied to the ethics committee of Yıldırım Beyazıt University in order to retrospectively examine the laboratory values in patients using canakinumab. The ethics committee found our research to be in line with ethical rules. Approval decision number: 2018/4/8. Consent was obtained from all patients before canakinumab treatment.

The study included pediatric cr-FMF patients who were receiving canakinumab treatment for $\geq 3$ months [canakinumab dose subcutaneous $2 \mathrm{mg} / \mathrm{kg}$, monthly (maximum dose $150 \mathrm{mg}$ )]. All patients fulfilled the clinical criteria for the diagnosis of FMF and had been on colchicine therapy for at least 3 months. The diagnostic criteria for FMF consist of the presence of at least two of the following characteristics: fever, abdominal pain, chest pain, arthritis, exertional leg pain and family history of FMF (1). Patients with FMF who were between the ages 8 -17 years were evaluated during the attack period. FMF attacks were confirmed by the presence of clinical findings such as fever, peritonitis, pleuritis, monoarthritis and high level of acute phase reactants. Colchicine resistance was defined as $\geq 1$ FMF attack per month for three consecutive months and/or a persistent elevation of one or more acute-phase reactants during the attack-free periods (including erythrocyte sedimentation rate, $\mathrm{C}$-reactive protein, fibrinogen and serum amyloid $\mathrm{A}$ ) and/or renal failure, although maximum colchicine dose was used for age and weight (10). Patients with systemic diseases other than familial Mediterranean fever and familial Mediterranean fever related complications were excluded from the study. Also, the patients with infection and with coincidental findings such as uveitis were not included in the study.

Age, gender, the duration of canakinumab treatment, MEFV gene mutations, complete blood count parameters,
ESR, CRP, SAA and fibrinogen levels were retrospectively collected from medical records of the patient files. All parameters were studied in our hospital laboratory. MPV, NLR, and PLR in the cr-FMF patients were measured prior to the first dose of canakinumab and at the time of administration of the last canakinumab dose. Blood samples of the patients were collected during attack-free intervals. The neutrophil/lymphocyte ratio (NLR) was determined by dividing the number of neutrophils by the number of lymphocytes, and the platelet/lymphocyte ratio PLR was determined by dividing the number of platelets by the number of lymphocytes.

The diagnosis of amyloidosis was confirmed by renal biopsy. All patients besides canakinumab treatment, continued to treat with colchicine.

\subsection{Statistical Analysis}

Statistical analysis was performed using SPSS Statistics for Windows software package, version 20.0 (SPSS Inc, Chicago, IL, USA). Continuous variables are presented as mean \pm SD and median, whereas categorical variables are shown as percentage. The Shapiro-Wilk test was used to determine the normality of the distribution of numerical variables. The independent samples $t$-test or the MannWhitney U-test was used for continuous variables. Spearman's rank nonparametric correlation analysis or Pearson correlation analysis was used for numerical variables. The paired samples $t$-test or Wilcoxon signed-rank test were used to compare the levels of inflammatory markers before and after canakinumab treatment. The level of statistical significance was set at $\mathrm{P}<0.05$.

\section{Results}

The study included 19 pediatric patients diagnosed as cr-FMF. In all, 19 (9 girls 47.4\%; 10 boys 52.6\%) cr-FMF patients receiving canakinumab treatment were evaluated, of which 3 had amyloidosis and nephrotic syndrome. Mean age of the patients was $11.5 \pm 4.7$ years (median 12 ). Mean age of diagnosis of patients was $4.5 \pm 2.7$ years (median 4; range 110 years). Mean time from diagnosis to the start of canakinumab treatment was $16.6 \pm 15.8$ months (median 8; minimum 3-maximum 55 months). Mean duration of disease before treatment with canakinumab was 5.6 \pm 4 years (median 5; range 1-12 years). Among the patients, 12 (63.1\%) had a family history of FMF and 6 (31.5\%) had consanguineous parents. Homozygous M694V mutation was detected in all cases.

The PLR was significantly lower after canakinumab treatment than before treatment (150.6 \pm 57.6 vs. $125.6 \pm$ $45.7, \mathrm{P}=0.003$ ) (Table 1 ). The pre-canakinumab treatment 
ESR, CRP and SAA levels were significantly higher than post treatment (64.6 \pm 33.3 vs. $22.9 \pm 19.6 \mathrm{~mm} / \mathrm{h}$ [P $<0.001], 66$ \pm 41.1 vs. $6.4 \pm 5.9 \mathrm{mg} / \mathrm{dL}[\mathrm{P}<0.001]$, and $22.3 \pm 36$ vs. $0.7 \pm$ $1.4 \mathrm{mg} / \mathrm{dL}$ [P $<0.001]$, respectively) (Table 1 ). The MPV and NLR did not differ significantly between before and after treatment with canakinumab $(7.8 \pm 1$ vs. $7.8 \pm 1.1 \mathrm{mg} / \mathrm{dL}$ $[\mathrm{P}=0.762]$ and $2.3 \pm 2.4$ vs. $1.7 \pm 1[\mathrm{P}=0.523]$, respectively (Table 1 ). In addition, the platelet count was significantly lower post canakinumab treatment (364.1 \pm 99.1 vs. 299.2 $\pm 98[\mathrm{P}=0.007]$ (Table 1 ).

Table 2 shows that the NLR and PLR were significantly lower post canakinumab treatment in the patients with FMF-related amyloidosis ( $8.1 \pm 4.7$ vs. $3.2 \pm 0.4[\mathrm{P}<0.001]$ and $149 \pm 79.7$ vs. $126.9 \pm 59.5$ [P $<0.001]$, respectively). There was no significant difference in the MPV, ESR, CRP, or SAA values before and after canakinumab treatment in the patients with FMF-related amyloidosis. Changes observed in inflammatory indices from pre to post canakinumab treatment in the patients with FMF-related amyloidosis are shown in Table 2.

The median duration of use of canakinumab was 26.6 (11-45) months). The effect of canakinumab on proteinuria was observed as a significant decrease in protein excretion in the patients with FMF-associated amyloidosis. The characteristics of FMF-associated amyloidosis patients are presented in Supplementary File Appendix 1.

There was a positive correlation between the PLR and $\operatorname{ESR}(r=0.115, P=0.047)$. The NLR exhibited a significant positive correlation with the CRP level $(r=0.604, P=0.006)$ and the ESR $(\mathrm{r}=0.722, \mathrm{P}<0.001)$. MPV was not correlated with the NLR $(r=-0.204, \mathrm{P}=0.401)$ or PLR $(r=-0.034, \mathrm{P}=$ $0.889)$, whereas there was a significantly positive correlation between the NLR and PLR $(r=0.475, P=0.040)$. Correlations between the MPV, NLR, and PLR, and the other parameters in the cr-FMF patients are shown in Table 3.

\section{Discussion}

The present study's findings show that the ESR, NLR, and PLR, CRP and SAA levels were affected by canakinumab treatment in children with cr-FMF, whereas MPV was not. Specifically, following canakinumab treatment the ESR, NLR, and PLR, CRP and SAA levels decreased. The observed decrease in the NLR was not significant, whereas the decrease observed in the ESR and PLR, and CRP SAA levels was significant.

Normally, pyrin suppresses neutrophil-mediated inflammation by reducing IL-1 synthesis, but MEFV gene mutations result in defective pyrin molecules. Mutated pyrin cannot prevent the progression of inflammation; thus, subclinical inflammation continues in patients with FMF, especially those with resistance to colchicine (11). High $\beta 2$ integrin and L-selectin levels on the neutrophil surface in FMF patients during episodes of exacerbation result in neutrophil adhesion to the endothelium and inflammation site (12). Consequently, an elevated NLR in patients FMF might be indicative of an increase in inflammation associated with neutrophils; thus, an elevated NLR may be associated with an increase in inflammation or FMF attacks $(4,13)$.

In present study all cr-FMF patients were receiving colchicine treatment. As a result of blocking the IL-1 pathway via colchicine therapy leukocytosis may not have occurred sufficiently, which might be why a significant decrease in the NLR following canakinumab therapy was not observed in the present study's patients (14). Ozer et al. (15) reported that the NLR, PLR, MPV, and RDW were higher in pediatric FMF patients during attack-free periods and that the NLR is the most reliable marker of subclinical inflammation (4). Other studies have reported that the NLR might be used as a marker of FMF attacks $(16,17)$.

FMF patient's systemic inflammation during attacks and chronic subclinical inflammation during attackfree periods might activate platelets and leukocytes (18). Platelet activation plays an important role in the pathogenesis of inflammation-induced thrombosis (19). Platelets store and secrete IL-1 $\beta$, and IL-1 may potentiate granulopoiesis and thrombopoiesis $(20,21)$. Hypercoagulability has been observed in FMF patients with major thrombotic events occurring with amyloidosis (22). Mice injected with IL-1 showed granulocyte/macrophage CSA, megakaryocyte CSA, and megakaryocyte potentiator (23). In the present study a significant decrease in the platelet count and the PLR was observed following canakinumab treatment, which is known to reduce thrombocytosis and subclinical inflammation via inhibition of IL- $1 \beta$; this might be why the platelet count and PLR decreased following canakinumab treatment in the present study. The decrease in the platelet count observed in the present study indicates that systemic inflammation persists in cr-FMF patients and that canakinumab treatment inhibits IL-1.

The present findings show that the PLR is positively correlated with the neutrophil count and inversely correlated with the lymphocyte count, further indicating that canakinumab affects granulopoiesis and thrombopoiesis via IL-1 blockade. Another indication of thrombocyte activation is platelet distribution width (PDW). In the present study there was a negative correlation between PDW, and the PLR and NLR.

As MPV is indicative of changes in platelet destruction and/or production, it is also an indicator of platelet volume and function (24). An inverse relationship between the platelet count and MPV is often indicative of a ten- 


\begin{tabular}{|c|c|c|c|}
\hline Variables & Before Canakinumab & After Canakinumab & P Value \\
\hline White blood cell, $\times 10^{3}$ cells $/ \mathrm{mm}^{3}$ & $7.9 \pm 2.4(7.8)$ & $7.3 \pm 2.4(7)$ & 0.077 \\
\hline Hemoglobin, g/dL & $11.9 \pm 1.7(12.1)$ & $12.8 \pm 2.0(12.3)$ & $0.015^{\mathrm{b}, \mathrm{c}}$ \\
\hline Platelet count, $\times 10^{3} / \mu \mathbf{L}$ & $364.1 \pm 99.1(365)$ & $299.2 \pm 98(273)$ & $0.007^{\mathrm{b}, \mathrm{d}}$ \\
\hline Mean platelet volume fl & $7.8 \pm 1(7.6)$ & $7.8 \pm 1.1(7.4)$ & 0.762 \\
\hline Platelet/lymphocyte ratio & $150.6 \pm 57.6(150.3)$ & $125.6 \pm 45.7(128.4)$ & $0.003^{\mathrm{b}, \mathrm{e}}$ \\
\hline Neutrophil count, $\times 10^{3}$ cells $/ \mathrm{mm}^{3}$ & $5.4 \pm 4.6(4.2)$ & $1.8 \pm 1(1.5)$ & 0.157 \\
\hline Lymphocyte count, $\times 10^{3}$ cells $/ \mathrm{mm}^{3}$ & $2.5 \pm 0.7(2.4)$ & $2.5 \pm 1.1(2.3)$ & 0.381 \\
\hline Neutrophil/lymphocyte ratio & $2.3 \pm 2.4(1.7)$ & $1.7 \pm 1(2.8)$ & 0.523 \\
\hline Monocytecount, $\times 10^{3} / \mu \mathrm{L}$ & $0.43 \pm 0.16(0.37)$ & $0.36 \pm 0.11(0.35)$ & 0.067 \\
\hline Eosinophil count, $\times 10^{3} / \mu \mathrm{L}$ & $0.13 \pm 0.08(0.11)$ & $0.13 \pm 0.09(0.11)$ & 0.192 \\
\hline Platelet distribution width, PDW fl & $45.1 \pm 8.2(44.9)$ & $43.4 \pm 13.6(43.9)$ & 0.850 \\
\hline Erythrocyte sedimentation rate, $\mathbf{m m} / \mathbf{h}$ & $64.6 \pm 33.3(57)$ & $22.9 \pm 19.6(18)$ & $<0.001^{\mathrm{b}, \mathrm{f}}$ \\
\hline C-reactive protein, $\mathrm{mg} / \mathrm{dL}$ & $66 \pm 41.1(54.8)$ & $6.4 \pm 5.9(3.1)$ & $<0.001^{\mathrm{b}, \mathrm{g}}$ \\
\hline Serum amyloid A protein, $\mathrm{mg} / \mathrm{dL}$ & $22.3 \pm 36(7.3)$ & $0.7 \pm 1.4(0.1)$ & $<0.001^{\mathrm{b}, \mathrm{h}}$ \\
\hline
\end{tabular}

${ }^{\mathrm{a}}$ Values are expressed as mean $\pm \mathrm{SD}$ (median).

${ }^{\mathrm{b}} \mathrm{P}<0.05$ is significant.

c $95 \%$ Confidence interval: upper -1.5 - lower -0.1.

${ }^{\mathrm{d}} 95 \%$ Confidence interval: upper 20.3 - lower 109.4.

e $95 \%$ Confidence interval: upper 1.0 - Lower 42.2.

${ }^{\mathrm{f}}$ 95\% Confidence interval: Upper 28.0 - lower 55.3.

${ }^{\mathrm{g}} 95 \%$ Confidence interval: upper 40.6 - lower 78.4

${ }^{\mathrm{h}} 95 \%$ Confidence interval: upper -5.5 - lower 64.5.

dency towards maintenance of a constant platelet mass and hemostasis (25). This inverse relationship is often observed in inflammatory disorders (26). In the present study canakinumab treatment had no effect on MPV. Published findings related to MPV in FMF patients are inconsistent. Some studies report that MPV does not change (as in the present study) $(27,28)$, some others report that MPV decreases in FMF patients (29), whereas a third group report that MPV increases in FMF patients $(15,16,30-33)$. Moreover, there was a positive correlation between PDW and MPV in the present study. Uluca et al. (9) also reported a positive correlation between MPV and PDW in FMF patients.

Makay et al. (34) reported that an increase in MPV is an important indicator of subclinical inflammation in pediatric FMF patients, especially those with proteinuria. Although MPV was observed to increase after canakinumab treatment in the present study's cr-FMF patients with amyloidosis, the increase was not significant. Uslu et al. (4) reported that the NLR was higher in FMF patients during attack-free periods and may be a useful marker for predicting the development of amyloidosis. In the present study the NLR was higher in the cr-FMF patients with FMFassociated amyloidosis than in those without amyloidosis. In addition, a significant decrease in the NLR and PLR was observed after canakinumab treatment in the patients with FMF-associated amyloidosis.

It is known that FMF patients have an elevated ESR, CRP and SAA levels (4), and that IL-1 $\beta$ inhibition via canakinumab treatment markedly decreases the plasma levels of IL-6 and high-sensitivity CRP (35).

The main limitation of our study was the retrospective design. Another limitation is the small number of patients, especially in the FMF-related amyloidosis group.

In the present study there was a significant decrease in the ESR, CRP and SAA levels after canakinumab treatment in cr-FMF patients. Moreoever, there was a strong positive correlation between the CRP level and ESR in the cr-FMF patients, but the CRP level and ESR were not affected by canakinumab treatment in the patients with FMF-related amyloidosis. Based on these findings, we think that the CRP level and ESR do not accurately indicate subclinical inflammation in cr-FMF patients with amyloidosis, which leads us to conclude that the PLR and NLR can be used as more reliable biomarkers in cr-FMF patients with amyloidosis than the CRP level and ESR.

\section{Supplementary Material}

Supplementary material(s) is available here [To read supplementary materials, please refer to the journal web- 


\begin{tabular}{|c|c|c|c|}
\hline Variables/Canakinumab & Mean \pm SD & Median & PValue \\
\hline MPV & & & 0.199 \\
\hline Before therapy & $7.3 \pm 0.5$ & 7.2 & \\
\hline After therapy & $7.7 \pm 0.9$ & 7.8 & \\
\hline NLR & & & $<0.001^{\mathrm{b}}$ \\
\hline Before therapy & $8.1 \pm 4.7$ & 8.1 & \\
\hline After therapy & $3.2 \pm 0.4$ & 3.2 & \\
\hline PLR & & & $<0.001^{\mathrm{b}}$ \\
\hline Before therapy & $149 \pm 79.7$ & 149 & \\
\hline After therapy & $126.9 \pm 59.5$ & 131.6 & \\
\hline ESR, $\mathbf{m m} / \mathbf{h}$ & & & 0.075 \\
\hline Before therapy & $114.3 \pm 25.5$ & 126 & \\
\hline After therapy & $54 \pm 22$ & 52 & \\
\hline CRP, mg/dL & & & 0.078 \\
\hline Before therapy & $60.9 \pm 33.4$ & 47 & \\
\hline After therapy & $8.3 \pm 6.8$ & 5.7 & \\
\hline $\mathrm{SAA}, \mathrm{mg} / \mathrm{dL}$ & & & 0.488 \\
\hline Before therapy & $4.5 \pm 5.8$ & 4.3 & \\
\hline After therapy & $0.09 \pm 0.01$ & 0.09 & \\
\hline
\end{tabular}

Abbreviations: CRP, C-reactive protein; ESR, erythrocyte sedimentation rate; MPV, Mean platelet volume; NLR, neutrophil/lymphocyte ratio; PLR, Platelet/lymphocyte ratio; SAA, Serum amyloid A.

${ }^{\mathrm{a}}$ The $95 \%$ confidence level was used.

${ }^{\mathrm{b}} \mathrm{P}<0.05$ is significant.

\begin{tabular}{|c|c|c|c|c|c|c|}
\hline \multirow[t]{2}{*}{ Variables } & \multicolumn{2}{|c|}{ MPV } & \multicolumn{2}{|c|}{ PLR } & \multicolumn{2}{|c|}{ NLR } \\
\hline & P Value & $\mathbf{r}$ & PValue & $\mathbf{r}$ & PValue & $\mathbf{r}$ \\
\hline Age & 0.503 & 0.164 & 0.440 & 0.188 & $0.050^{\mathrm{a}}$ & 0.455 \\
\hline Gender & 0.783 & -0.068 & 0.652 & -0.111 & 0.815 & 0.058 \\
\hline White blood cells, $\times \mathbf{1 0}^{3}$ cells $/ \mathrm{mm}^{3}$ & 0.053 & -0.401 & 0.858 & 0.044 & 0.192 & 0.313 \\
\hline Hemoglobin, $g / d L$ & 0.671 & -0.104 & 0.911 & 0.028 & $0.049^{\mathrm{a}}$ & -0.353 \\
\hline Platelet count, $\mathbf{v i o}^{3} / \mu \mathbf{L}$ & $0.013^{\mathrm{a}}$ & -0.568 & 0.075 & 0.418 & 0.947 & -0.016 \\
\hline Neutrophil count, $\times 10^{3}$ cells $/ \mathrm{mm}^{3}$ & 0.889 & -0.034 & $0.040^{\mathrm{a}}$ & 0.475 & $0.000^{\mathrm{a}}$ & 0.768 \\
\hline Lymphocyte count, $\times 10^{3}$ cells $/ \mathrm{mm}^{3}$ & 0.278 & -0.262 & $0.019^{\mathrm{a}}$ & -0.511 & 0.070 & -0.475 \\
\hline Monocyte count, $\times 10^{3} / \mu \mathrm{L}$ & 0.130 & -0.371 & $0.046^{\mathrm{a}}$ & 0.096 & 0.542 & 0.154 \\
\hline Eosinophil count, $\times 10^{3} / \mu \mathrm{L}$ & 0.242 & -0.291 & 0.944 & -0.018 & 0.272 & 0.275 \\
\hline Platelet distribution width, PDW fl & $0.018^{\mathrm{a}}$ & 0.550 & $0.005^{\mathrm{a}}$ & -0.626 & $0.044^{\mathrm{a}}$ & -0.080 \\
\hline Erythrocyte sedimentation rate, $\mathbf{m m} / \mathbf{h}$ & 0.964 & 0.011 & $0.047^{\mathrm{a}}$ & 0.115 & $0.000^{\mathrm{a}}$ & 0.722 \\
\hline C-reactive protein, $\mathrm{mg} / \mathrm{dL}$ & 0.491 & 0.168 & 0.715 & 0.090 & $0.006^{\mathrm{a}}$ & 0.604 \\
\hline Serum amyloid A protein, mg/dL & 0.134 & 0.458 & 0.513 & 0.210 & 0.924 & 0.031 \\
\hline Mean platelet volume fl & & & 0.401 & -0.204 & 0.889 & -0.034 \\
\hline Platelet/lymphocyte ratio & 0.401 & -0.204 & & & $0.040^{\mathrm{a}}$ & 0.475 \\
\hline Neutrophil/lymphocyte ratio & 0.889 & -0.034 & $0.040^{*}$ & 0.475 & & \\
\hline
\end{tabular}

Abbreviations: MPV, mean platelet volume; NLR, neutrophil/lymphocyte ratio; PLR, platelet/lymphocyte ratio; Can: Canakinumab.

${ }^{\mathrm{a}} \mathrm{P}<0.05$ is significant. 
site and open PDF/HTML].

\section{Footnotes}

Authors' Contribution: Study conception and design: Fatma Yazılıtaș. Acquisition of data: contribution of all author's. Analysis and interpretation of data: Fatma Yazılıtas and Semanur Özdel. Drafting of the manuscript: Fatma Yazılıtaș and Semanur Özdel. Critical revision: contribution of all author's.

Conflict of Interests: It is not declared by the authors.

Ethical Approval: This article does not contain any studies with human participants or animals performed by any of the authors. no human was used as a subject in the study. The study was prepared by examining the files retrospectively.

Ethical Considerations: This work was approved by the the Ethics Committee of Yıldırım Beyazıt University. Approval decision number: 2018/4/8.

Financial Disclosure: No financial disclosure.

Funding/Support: No funding.

Patient Consent: Consent was obtained from all patients before canakinumab treatment.

\section{References}

1. Ozen S, Bilginer Y. A clinical guide to autoinflammatory diseases: Familial Mediterranean fever and next-of-kin. Nat Rev Rheumatol. 2014;10(3):135-47. doi:10.1038/nrrheum.2013.174. [PubMed: 24247370].

2. Chae JJ, Wood G, Richard K, Jaffe H, Colburn NT, Masters SL, et al. The familial Mediterranean fever protein, pyrin, is cleaved by caspase1 and activates NF-kappaB through its N-terminal fragment. Blood. 2008;112(5):1794-803. doi: 10.1182/blood-2008-01-134932. [PubMed 18577712]. [PubMed Central: PMC2518886].

3. Grattagliano I, Bonfrate L, Ruggiero V, Scaccianoce G, Palasciano G, Portincasa P. Novel therapeutics for the treatment of familial Mediterranean fever: From colchicine to biologics. Clin Pharmacol Ther. 2014;95(1):89-97. doi: 10.1038/clpt.2013.148. [PubMed: 23867542].

4. Uslu AU, Deveci K, Korkmaz S, Aydin B, Senel S, Sancakdar E, et al. Is neutrophil/lymphocyte ratio associated with subclinical inflammation and amyloidosis in patients with familial Mediterranean fever? Biomed Res Int. 2013;2013:185317. doi: 10.1155/2013/185317. [PubMed: 23865042]. [PubMed Central: PMC3705820].

5. Bilginer Y, Akpolat T, Ozen S. Renal amyloidosis in children. Pediatr Nephrol. 2011;26(8):1215-27. doi: 10.1007/s00467-011-1797-x. [PubMed: 21360109]. [PubMed Central: PMC3119800].

6. Haviv R, Hashkes PJ. Canakinumab investigated for treating familial Mediterranean fever. Expert Opin Biol Ther. 2016;16(11):1425-34. doi: 10.1080/14712598.2016.1233963. [PubMed: 27603969].

7. Balta S, Celik T, Mikhailidis DP, Ozturk C, Demirkol S, Aparci M, et al. The relation between atherosclerosis and the neutrophillymphocyte ratio. Clin Appl Thromb Hemost. 2016;22(5):405-11. doi: 10.1177/1076029615569568. [PubMed: 25667237].

8. Templeton AJ, Ace O, McNamara MG, Al-Mubarak M, Vera-Badillo FE, Hermanns T, et al. Prognostic role of platelet to lymphocyte ratio in solid tumors: A systematic review and meta-analysis. CancerEpidemiol
Biomarkers Prev. 2014;23(7):1204-12. doi: 10.1158/1055-9965.EPI-14-0146. [PubMed: 24793958].

9. Uluca U, Ece A, Sen V, Karabel D, Yel S, Gunes A, et al. Usefulness of mean platelet volume and neutrophil-to-lymphocyte ratio for evaluation of children with Familial Mediterranean fever Med Sci Monit. 2014;20:1578-82. doi: 10.12659/MSM.892139. [PubMed: 25189760]. [PubMed Central: PMC4165509].

10. Ozen S, Kone-Paut I, Gul A. Colchicine resistance and intolerance in familial mediterranean fever: Definition, causes, and alternative treatments. Semin Arthritis Rheum. 2017;47(1):115-20. doi: 10.1016/j.semarthrit.2017.03.006. [PubMed: 28413100].

11. Yildirim K, Uzkeser H, Keles M, Karatay S, Kiziltunc A, Kaya MD, et al Relationship between serum interleukin-1beta levels and acute phase response proteins in patients with familial Mediterranean fever. Biochem Med (Zagreb). 2012;22(1):109-13. doi: 10.11613/BM.2012.012. [PubMed: 22384525]. [PubMed Central: PMC4062326].

12. Molad Y, Fridenberg A, Bloch K, Langevitz P, Mukamel M, Sulkes J, et al. Neutrophil adhesion molecule expression in familial Mediterranean fever: Discordance between the intravascular regulation of beta2 integrin and L-selectin expression in acute attack. J Investig Med 2004;52(1):58-61. doi:10.1136/jim-52-01-28. [PubMed: 14989371].

13. Ahsen A, Ulu MS, Yuksel S, Demir K, Uysal M, Erdogan M, et al. As a new inflammatory marker for familial Mediterranean fever: Neutrophil-to-lymphocyte ratio. Inflammation. 2013;36(6):135762. doi: 10.1007/s10753-013-9675-2. [PubMed: 23794006].

14. Ben-Chetrit E, Levy M. Colchicine: 1998 update. Semin Arthritis Rheum. 1998;28(1):48-59. doi: 10.1016/S0049-0172(98)80028-0. [PubMed: 9726336].

15. Ozer S, Yilmaz R, Sonmezgoz E, Karaaslan E, Taskin S, Butun I, et al. Simple markers for subclinical inflammation in patients with Familial Mediterranean Fever. Med Sci Monit. 2015;21:298-303. doi: 10.12659/MSM.892289. [PubMed: 25615955]. [PubMed Central: PMC4315639].

16. Basaran O, Uncu N, Celikel BA, Aydin F, Cakar N. Assessment of neutrophil to lymphocyte ratio and mean platelet volume in pediatric familial Mediterranean fever patients. J Res Med Sci. 2017;22:35. doi: 10.4103/1735-1995.202140. [PubMed: 28461821]. [PubMed Central: PMC5390538].

17. Celikbilek M, Dogan S, Akyol L, Borekci E, Zararsiz G, Kozan M, et al. Neutrophil-lymphocyte ratio in patients with familial Mediterranean fever. J Clin Lab Anal. 2015;29(1):80-3. doi: 10.1002/jcla.21732. [PubMed: 24687426]

18. Ben-Zvi I, Livneh A. Chronic inflammation in FMF: Markers, risk factors, outcomes and therapy. Nat Rev Rheumatol. 2011;7(2):105-12. doi 10.1038/nrrheum.2010.181. [PubMed: 21060333].

19. Esmon CT. Inflammation and thrombosis. J Thromb Haemost. 2003;1(7):1343-8. doi: 10.1046/j.1538-7836.2003.00261.x. [PubMed: 12871267].

20. Yang M, Li K, Chui CM, Yuen PM, Chan PK, Chuen CK, et al. Expression of interleukin (IL)1 type I and type II receptors in megakaryocytic cells and enhancing effects of IL-1beta on megakaryocytopoiesis and NF-E2 expression. Br J Haematol. 2000;111(1):371-80. doi: 10.1046/j.1365 2141.2000.02340.x. [PubMed: 11091227].

21. Chuen CK, Li K, Yang M, Fok TF, Li CK, Chui CM, et al. Interleukin-1beta up-regulates the expression of thrombopoietin and transcription factors c-Jun, c-Fos, GATA-1, and NF-E2 in megakaryocytic cells. J Lab Clin Med. 2004;143(2):75-88. doi: 10.1016/j.lab.2003.09.006. [PubMed: 14966463].

22. La Regina M, Orlandini F, Manna R. Autoinflammatory diseases: A possible cause of thrombosis? ThrombJ.2015;13:19. doi:10.1186/s12959-0150049-x. [PubMed: 25969671]. [PubMed Central: PMC4428094].

23. Nakai S, Aihara K, Hirai Y. Interleukin-1 potentiates granulopoiesis and thrombopoiesis by producing hematopoietic factors in vivo. Life Sci. 1989;45(7):585-91. doi: 10.1016/0024-3205(89)90043-x. [PubMed 2671565]. 
24. Jagroop IA, Clatworthy I, Lewin J, Mikhailidis DP. Shape change in human platelets: Measurement with a channelyzer and visualisation by electron microscopy. Platelets. 2000;11(1):28-32. doi: 10.1080/09537100075760. [PubMed: 10938878].

25. Thompson CB. From precursor to product: How do megakaryocytes produce platelets? Prog Clin Biol Res. 1986;215:361-71. [PubMed: 3523524].

26. Aksu K, Donmez A, Keser G. Inflammation-induced thrombosis: Mechanisms, disease associations and management. Curr Pharm Des. 2012;18(11):1478-93. doi: 10.2174/138161212799504731. [PubMed: 22364132].

27. Gasparyan AY, Ayvazyan L, Mikhailidis DP, Kitas GD. Mean platelet volume: A link between thrombosis and inflammation? Curr Pharm Des. 2011;17(1):47-58. doi: 10.2174/138161211795049804. [PubMed: 21247392].

28. Demirel A, Celkan T, Kasapcopur O, Bilgen H, Ozkan A, Apak H, et al. Is Familial Mediterranean Fever a thrombotic disease or not? Eur J Pediatr. 2008;167(3):279-85. doi: 10.1007/s00431-007-0475-2. [PubMed: 17436016].

29. Uluca U, Demir F, Ece A, Sen V, Gunes A, Aktar F, et al. Assessment of epicardial adipose tissue thickness and the mean platelet volume in children with familial Mediterranean fever. Ital J Pediatr. 2015;41:15. doi: 10.1186/s13052-015-0120-z. [PubMed: 25887962]. [PubMed Central: PMC4348162].

30. Sahin S, Senel S, Ataseven H, Yalcin I. Does mean platelet vol- ume influence the attack or attack-free period in the patients with Familial Mediterranean fever? Platelets. 2013;24(4):320-3. doi: 10.3109/09537104.2012.697591. [PubMed: 22720903].

31. Arica S, Ozer C, Arica V, Karakus A, Celik T, Gunesacar R. Evaluation of the mean platelet volume in children with familial Mediterranean fever. Rheumatol Int. 2012;32(11):3559-63. doi: 10.1007/s00296-011-2251x. [PubMed: 22086472].

32. Dursun I, Gok F, Babacan O, Sarı E, Sakallıoglu O. Are mean platelet volume and splenomegaly subclinical inflammatory marker in children with familial mediterranean fever? Health. 2010;2(7):692-5. doi: 10.4236/health.2010.27105.

33. Karakurt Ariturk O, Ureten K, Sari M, Yazihan N, Ermis E, Erguder I. Relationship of paraoxonase-1, malondialdehyde and mean platelet volume with markers of atherosclerosis in familial Mediterranean fever: An observational study. Anadolu Kardiyol Derg. 2013;13(4):357-62. doi: 10.5152/akd.2013.103. [PubMed: 23531873].

34. Makay B, Turkyilmaz Z, Unsal E. Mean platelet volume in children with familial Mediterranean fever. Clin Rheumatol. 2009;28(8):975-8. doi: 10.1007/s10067-009-1148-5. [PubMed: 19283330].

35. Sakalli H, Kal O. Mean platelet volume as a potential predictor of proteinuria and amyloidosis in familial Mediterranean fever. Clin Rheumatol. 2013;32(8):1185-90. doi: 10.1007/s10067-013-2257-8. [PubMed: 23592230]. 